\title{
Cartographie des débits d'étiage : application au bassin de la Meurthe
}

Cartography of low water conditions: application to the Meurthe basin Kartographie der Abflüsse bei Niedrigwasser : Anwendung auf das Becken der Meurthe

Isabelle Arts et Michel Sary

\section{(2) OpenEdition}

\section{Édition électronique}

URL : http://journals.openedition.org/rge/4175

DOI : 10.4000/rge.4175

ISSN : 2108-6478

Éditeur

Association des géographes de l'Est

Édition imprimée

Date de publication : 1 janvier 2000

ISSN : 0035-3213

Référence électronique

Isabelle Arts et Michel Sary, "Cartographie des débits d'étiage : application au bassin de la Meurthe », Revue Géographique de l'Est [En ligne], vol. 40 / 1-2 | 2000, mis en ligne le 05 août 2013, consulté le 10 décembre 2020. URL : http://journals.openedition.org/rge/4175; DOI : https://doi.org/10.4000/rge. 4175

Ce document a été généré automatiquement le 10 décembre 2020.

Tous droits réservés 


\title{
Cartographie des débits d'étiage : application au bassin de la Meurthe
}

\author{
Cartography of low water conditions: application to the Meurthe basin \\ Kartographie der Abflüsse bei Niedrigwasser : Anwendung auf das Becken der \\ Meurthe
}

Isabelle Arts et Michel Sary

1 Depuis plusieurs années, le Laboratoire de Géographie Physique du CEGUM mène des recherches sur les débits d'étiage dans le bassin Rhin-Meuse. Ces recherches s'inscrivent dans un programme d'étude générale qui a pour but la connaissance précise de la ressource en eau dans le temps et dans l'espace, afin d'aboutir à terme à une gestion équilibrée de cette ressource et de ses différentes utilisations. Ce programme a permis l'élaboration d'une méthodologie pour l'étude des débits d'étiage (François et al., 1994), méthodologie qui a été appliquée pour la réalisation du catalogue des débits caractéristiques des cours d'eau du bassin de la Meurthe (Arts et al., 1996). Les données de ce catalogue font actuellement l'objet d'une étude méthodologique pour la cartographie des écoulements des bassins de la Meuse et de la Moselle françaises (Arts et François, 1997) dont les résultats intermédiaires, concernant le bassin de la Meurthe, sont présentés ici.

\section{Le bassin de la Meurthe}

2 C'est un bassin au comportement hydrologique diversifié qui est bien équipé en stations hydrométriques et qui a fait l'objet de nombreuses campagnes de jaugeages en basses eaux.

3 La superficie du bassin de la Meurthe est de $3085 \mathrm{~km} 2$. La rivière prend sa source sur le flanc nord-ouest du massif du Hohneck (à 1075 m d'altitude) et conflue avec la Moselle à Frouard (à $190 \mathrm{~m}$ d'altitude), $157 \mathrm{~km}$ en aval. Son cours, orienté sud-est/nord-ouest, traverse deux secteurs géographiques bien différenciés (Fig. 1). 
Fig. 1 : Bassin de la Meurthe : carte de présentation

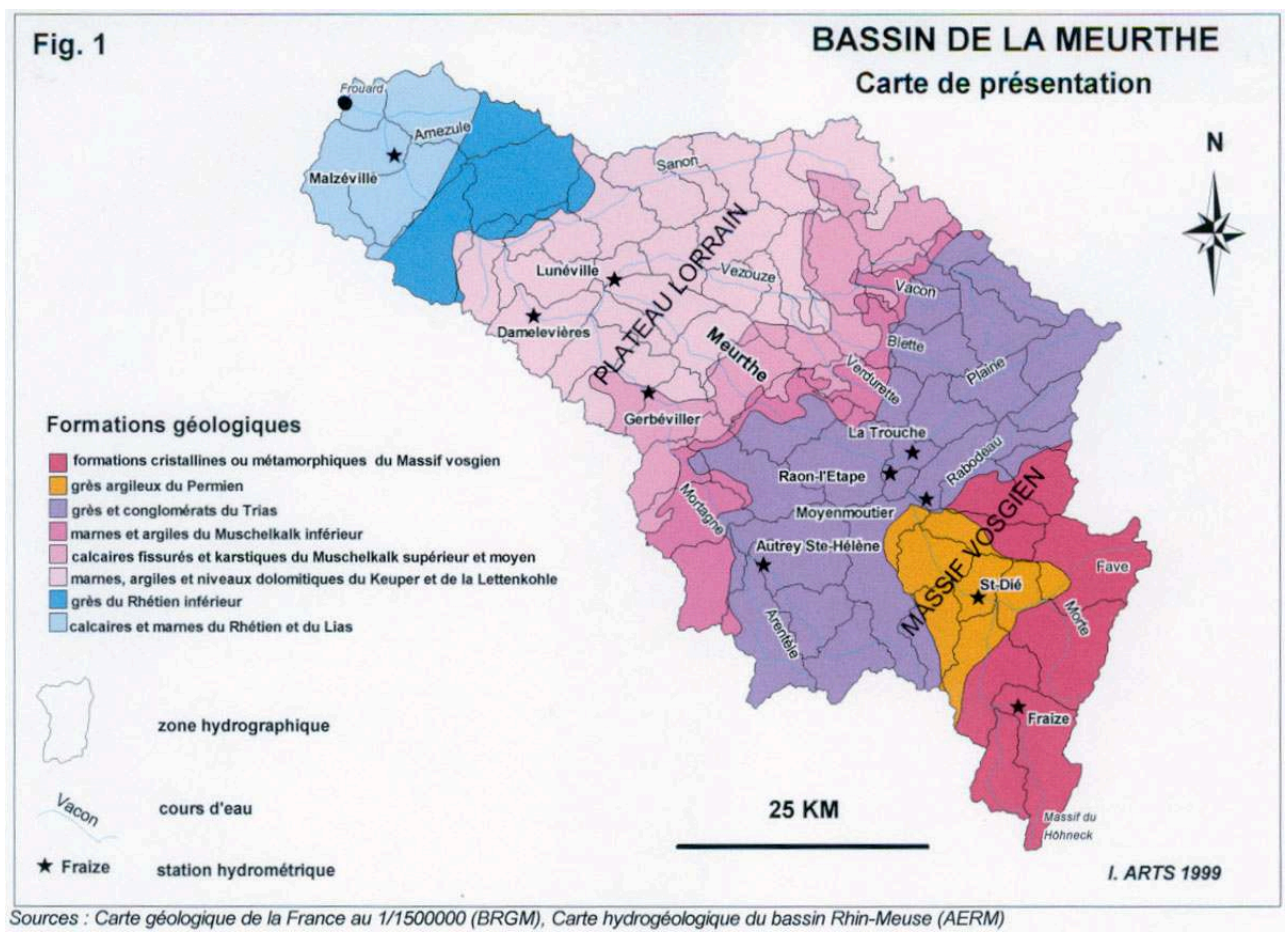

\section{A. Les différents milieux naturels}

4 Au sud-est, le Massif vosgien, avec des altitudes dépassant parfois $1300 \mathrm{~m}$, offre des potentialités aquifères importantes. Les formations cristallines très fracturées, avec de nombreuses zones d'altération (arènes granitiques), constituent un réservoir morcelé, mais bien alimenté par des précipitations importantes (plus de $2000 \mathrm{~mm}$ par an sur les hautes crêtes). Les grès du Trias, avec plus de $100 \mathrm{~m}$ d'épaisseur et une porosité d'interstices élevée, présentent un réservoir plus homogène, bien alimenté par des précipitations de plus de $1000 \mathrm{~mm}$ par an. Entre ces deux formations, les grès argileux du Permien ne constituent qu'un faible aquifère.

5 Au nord-ouest, la partie du Plateau lorrain drainée par la Meurthe ne bénéficie pas des mêmes conditions d'alimentation pluviométrique et ne comprend pas d'aquifères puissants. En effet, ceux-ci sont situés dans des séries sédimentaires composées essentiellement de calcaires et de marnes, où seuls les calcaires du Muschelkalk et les grès du Rhétien peuvent localement soutenir les étiages. D'autre part, les altitudes faibles ( $400 \mathrm{~m}$ au maximum au nord du bassin) ainsi que la relative position d'abri par rapport au relief de côte de direction méridienne, situé à l'ouest, entraînent des précipitations moins abondantes (700 à $800 \mathrm{~mm}$ ).

6 Ces différences d'alimentation pluviométrique et de potentialité aquifère du bassin de la Meurthe induisent des écoulements bien différenciés, surtout en période d'étiage où ceux-ci sont uniquement alimentés par les nappes souterraines. 


\section{B. Les données hydrométriques}

7 Ces données sont obtenues par les enregistrements des stations hydrométriques et par des campagnes de jaugeages en période de basses eaux :

- les stations hydrométriques permettent de saisir l'évolution des écoulements dans le temps. Pour cette étude, 10 stations ont été retenues ( 5 sur la Meurthe et 5 sur les principaux affluents, tableau 1) et traitées sur la période 1971-1990, les lacunes de certaines d'entre elles ayant été comblées par simple corrélation. Ces stations sont actuellement gérées par la DIREN Lorraine ;

9 - les campagnes de jaugeages ont été organisées en période de basses eaux, en plusieurs étapes de 1978 à 1996 pour différentes situations hydrologiques (étiage plus ou moins prononcé). Les mesures de débit ont été effectuées sur la Meurthe et les affluents de différents ordres, pour des surfaces partielles de bassin de 5 à $10 \mathrm{~km} 2$, soit environ 500 points mesurés utilisés dans cette étude.

Tableau 1 : Stations hydrométriques retenues dans le bassin de la Meurthe (cours d'eau principal et affluents)

\begin{tabular}{|l|l|c|c|c|c|}
\hline \multicolumn{1}{|c|}{ Station } & \multicolumn{1}{|c|}{ Cours d'eau } & Code hydro & Surface en $\mathrm{km}^{2}$ & PKH & $\begin{array}{c}\text { Date de mise } \\
\text { en service }\end{array}$ \\
\hline Fraize & Meurthe & A 600010 A & 69 & 861,04 & 1971 \\
\hline Saint-Dié & Meurthe & A 605010 A & 369 & 880,37 & 1967 \\
\hline Moyenmoutier & Rabodeau & A 614020 A & 151 & 998,6 & $\begin{array}{c}1969 \\
\text { (a fonctionné } \\
\text { jusqu'en 1984) }\end{array}$ \\
\hline Raon L'Étape & Meurthe & A 615010 A & 727 & 901,54 & 1973 \\
\hline La Trouche & Plaine & A 623 020 A & 116 & 996,00 & 1970 \\
\hline Lunéville & Vezouze & A 657 011 A & 559 & 997,62 & 1969 \\
\hline $\begin{array}{l}\text { Autrey } \\
\text { Sainte-Hélène }\end{array}$ & Mortagne & A 662 012 A & 98 & 947,25 & 1969 \\
\hline Gerbéviller & Mortagne & A 673 012 A & 495 & 983,75 & 1970 \\
\hline Damelevières & Meurthe & A 676 010 A & 2288 & 965,76 & 1969 \\
\hline Malzéville & Meurthe & A 694 010 A & 2914 & 990,91 & 1959 \\
\hline
\end{tabular}

10 Ainsi, les deux types de données hydrométriques sont complémentaires. Les stations fournissent des données en continu, mais elles ne caractérisent que l'exutoire du bassin qu'elles contrôlent (globalisation des écoulements internes du bassin). Les campagnes de jaugeages permettent une investigation spatiale des différents écoulements à l'intérieur du bassin, mais chaque campagne n'est représentative que d'une situation hydrologique particulière.

\section{Les profils hydrologiques}

11 Les débits des campagnes de jaugeages en basses eaux permettent l'élaboration des profils hydrologiques des différents cours d'eau. 


\section{A. L'élaboration des profils hydrologiques}

débit spécifique de la zone homogène de bassin versant relative à ce segment :

- si la pente est égale à 1 , le débit spécifique est identique pour toute la zone homogène (débit et surface augmentent de la même façon),

- si elle est supérieure à 1 , le débit spécifique augmente de manière exponentielle (le débit augmente plus rapidement que la surface),

- si elle est inférieure à 1 , le débit spécifique diminue de la même manière (la surface augmente plus rapidement que le débit).

\section{B. L'exemple de la Mortagne}

La Mortagne conflue avec la Meurthe, en rive gauche de celle-ci, entre les stations hydrométriques de Lunéville et de Damelevières. Son bassin versant, dont la superficie est de $582 \mathrm{~km} 2$, s'inscrit dans les formations gréseuses du Trias à l'amont (jusqu'à la station d'Autrey Sainte-Hélène) et dans les formations calcaires et argileuses du Muschelkalk et du Keuper à l'aval (Fig. 1).

De nombreuses campagnes de jaugeages ont été réalisées sur ce cours d'eau depuis une quinzaine d'années. Si l'on prend par exemple celles de 1989 (Fig. 2), on se rend compte d'une part que les débits s'alignent pratiquement sur des segments de droite et d'autre part que ces segments sont parallèles d'une campagne de mesures à l'autre. Sur le graphique, on constate qu'il existe une "cassure " des trois profils à une surface d'environ $60 \mathrm{~km} 2$, ce qui correspond à peu près à la zone où la Mortagne quitte les grès vosgiens pour s'écouler sur les calcaires du Plateau lorrain. On peut donc expliquer les pentes (g) plus fortes à l'amont par la présence d'aquifères puissants (grès) qui entrainent des rendements élevés. À l'aval, où les pentes sont plus faibles (calcaires et argiles), les légères variations de pente sont dues à la présence de petits affluents qui entraînent localement une augmentation de débit. 
Fig. 2 : Profils hydrologiques de la Mortagne

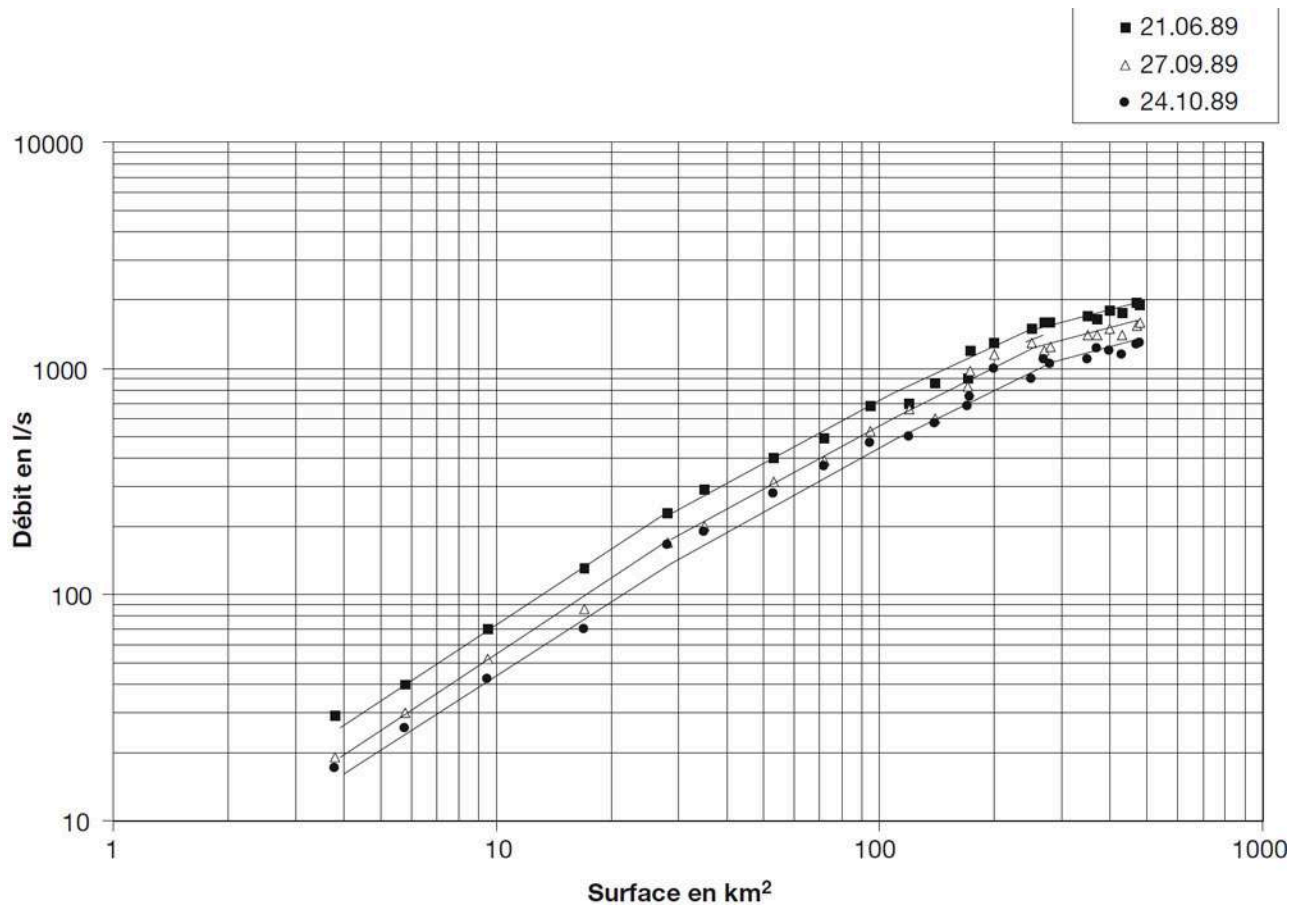

\section{Les autres cours d'eau}

Les quatre cours d'eau représentés (Fig. 3) montrent la variété des profils hydrologiques des affluents.

Fig. 3 : Profils hydrologiques de divers affluents

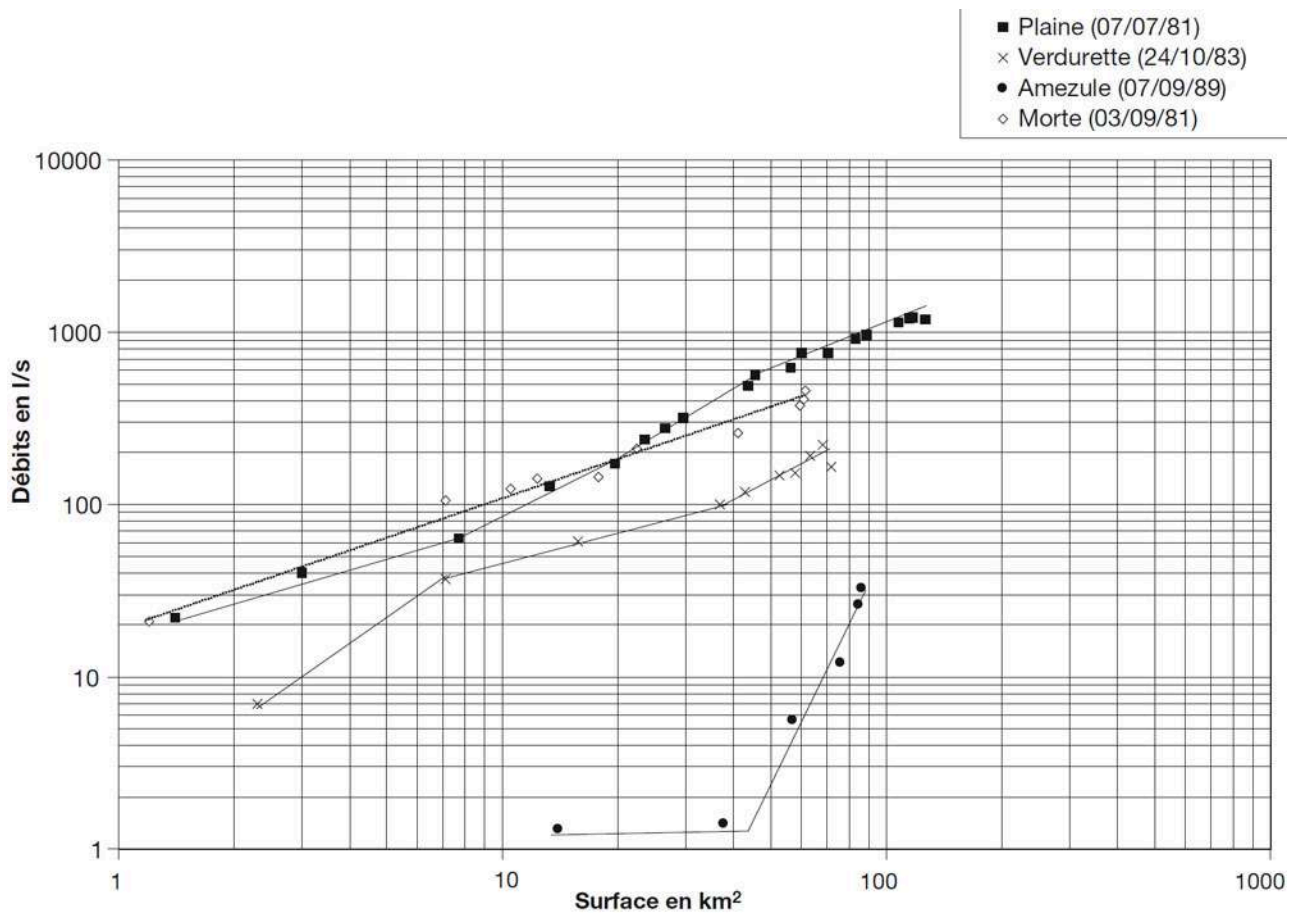


21 La Plaine (surface de bassin : $127 \mathrm{~km} 2$ ), affluent de rive droite de la Meurthe en aval de Raon l'Étape, s'écoule intégralement dans les grès du Trias. Les pentes du profil hydrologique diffèrent peu et sont proches de 1 , ce qui traduit une alimentation régulière tout au long du cours d'eau.

La Verdurette (surface de bassin : $73 \mathrm{~km} 2$ ) est un affluent de rive gauche de la Vezouze. Elle prend sa source dans les grès du Trias (d'où une pente supérieure à 1 à l'amont). Ensuite, elle s'écoule successivement dans les marnes et argiles du Muschelkalk (pente proche de 0,5) puis dans les calcaires du Muschelkalk (pente à nouveau proche de 1).

23 L'Amezule (surface de bassin : $87 \mathrm{~km} 2$ ) est un affluent de rive droite de la Meurthe, à l'extrémité aval du bassin. Elle s'écoule d'abord dans les marnes du Lias inférieur (débits d'étiage très faibles et pente quasi nulle à l'amont) puis dans les formations calcaro-gréseuses du Lias supérieur (pente proche de 10).

24 La Morte (surface de bassin : $62 \mathrm{~km} 2$ ) est un affluent de rive gauche de la Fave. Elle s'inscrit presque intégralement dans les gneiss de Sainte-Marie-aux-Mines et, de ce fait, son profil hydrologique ne comporte qu'un seul segment (pente égale à 0,7 ).

On constate donc, même si la Meurthe demeure un bassin relativement homogène dans son ensemble, que les cours d'eau ont des profils hydrologiques variés. L'allure de chaque profil est représentative des conditions hydrogéologiques des différents milieux du bassin que recoupe le cours d'eau.

\section{Les débits caractéristiques}

Les débits d'étiage ont été calculés à chaque station pour les fréquences caractéristiques $1 / 2,1 / 5$ et $1 / 10$ (tableau 2 ) en utilisant la loi log-normale. Les valeurs de ces débits et celle du module sont exprimées en $1 / \mathrm{s} / \mathrm{km} 2$ pour apprécier le rendement hydrologique des différents bassins.

Tableau 2 : Débits caractéristiques des stations hydrométriques (période 1971-1990)

\begin{tabular}{|c|c|c|c|c|c|c|c|c|c|c|}
\hline \multirow{3}{*}{ Station } & \multirow{3}{*}{$\begin{array}{l}\text { Cours } \\
\text { d'eau }\end{array}$} & \multirow{3}{*}{$\begin{array}{c}\text { Surface } \\
\left(\mathrm{km}^{2}\right)\end{array}$} & \multicolumn{8}{|c|}{ Débits mensuels d'étiage fréquentiel } \\
\hline & & & Module & Q 1/2 & Q 1/5 & Q 1/10 & Module & Q 1/2 & Q 1/5 & Q $1 / 10$ \\
\hline & & & $1 / 5$ & $1 / s$ & $1 / s$ & $1 / s$ & $\mathrm{l} / \mathrm{s} / \mathrm{km}^{2}$ & $1 / \mathrm{s} / \mathrm{km}^{2}$ & $\mathrm{l} / \mathrm{s} / \mathrm{km}^{2}$ & $\mathrm{l} / \mathrm{s} / \mathrm{km}^{2}$ \\
\hline Fraize & Meurthe & 69 & 2110 & 743 & 563 & 488 & 30,6 & 10,8 & 8,2 & 7,1 \\
\hline Saint-Dié & Meurthe & 369 & 7891 & 2187 & 1559 & 1307 & 21,4 & 5,9 & 4,2 & 3,5 \\
\hline Moyenmoutier & Rabodeau & 151 & 2673 & 1280 & 956 & 821 & 17,7 & 8,5 & 6,3 & 5,4 \\
\hline Raon l'Étape & Meurthe & 727 & 14840 & 4373 & 3109 & 2603 & 20,4 & 6 & 4,3 & 3,6 \\
\hline La Trouche & Plaine & 116 & 2318 & 895 & 644 & 543 & 20 & 7,7 & 5,6 & 4,7 \\
\hline Lunéville & Vezouze & 559 & 6538 & 1439 & 1026 & 860 & 11,7 & 2,6 & 1,8 & 1,5 \\
\hline Autrey & Mortagne & 98 & 1708 & 691 & 502 & 425 & 17,4 & 7,1 & 5,1 & 4,3 \\
\hline Gerbéviller & Mortagne & 495 & 5842 & 1796 & 1208 & 982 & 11,8 & 3,6 & 2,4 & 2 \\
\hline Damelevières & Meurthe & 2288 & 34070 & 8861 & 6213 & 5165 & 14,9 & 3,9 & 2,7 & 2,3 \\
\hline Malzéville & Meurthe & 2914 & 39660 & 9892 & 7054 & 5916 & 13,6 & 3,4 & 2,4 & 2 \\
\hline
\end{tabular}

\section{A. La différenciation des rendements hydrologiques}

Globalement, les rendements sont plus élevés pour les stations du Massif vosgien (Fraize, Saint-Dié, Moyen-moutier, Raon l'Étape, la Trouche et Autrey Sainte-Hélène) que pour celles du Plateau lorrain (Lunéville, Gerbéviller, Damelevières et Malzéville) : 
- pour le module, les rendements sont de l'ordre de 18 à $21 \mathrm{l} / \mathrm{s} / \mathrm{km} 2$ pour les bassins du Massif vosgien et de 12 à $15 \mathrm{l} / \mathrm{s} / \mathrm{km} 2$ pour les bassins du Plateau lorrain, soit une diminution d'environ un tiers. Ceci s'explique par des précipitations plus élevées sur le massif que sur le plateau ;

- pour les débits d'étiage aux fréquences caractéristiques, les bassins du Massif vosgien ont également des rendements élevés $(\mathrm{Q} 1 / 2: 6$ à $8 \mathrm{l} / \mathrm{s} / \mathrm{km} 2, \mathrm{Q} 1 / 5: 4$ à 6 et $\mathrm{Q} 1 / 10: 3$ à 5) alors que les rendements des bassins du Plateau lorrain sont plus faibles $(\mathrm{Q} 1 / 2: 3$ à 4 $1 / \mathrm{s} / \mathrm{km} 2, \mathrm{Q} 1 / 5: 2$ à $3, \mathrm{Q} 1 / 10: 1.5$ à 2 ). Cette diminution de rendement de moitié entre les deux milieux est due essentiellement à la nature différente des aquifères. Les rendements des bassins du plateau, et plus particulièrement le bassin de la Meurthe à Damelevières et à Malzéville, seraient encore plus faibles si ceux-ci ne bénéficiaient pas des apports des bassins amont du Massif vosgien.

Ainsi, que ce soit pour le module où le ruissellement joue un rôle important, ou que ce soit pour les débits d'étiage où la restitution des nappes est pratiquement exclusive, la différence entre le Massif vosgien et le Plateau lorrain est très bien marquée.

\section{B. L'évolution des rendements hydrologiques par station}

31 L'évolution des rendements est appréciée par l'analyse des rapports successifs des débits caractéristiques pour chaque station (tableau 3).

Tableau 3 : Rapports entre les débits par station

\begin{tabular}{|c|c|c|c|}
\hline Station & Q 1/2 module & Q 1/5Q 1/2 & Q 1/10/Q 1/5 \\
\hline Fraize & $35 \%$ & $76 \%$ & $87 \%$ \\
\hline Saint-Dié & $28 \%$ & $71 \%$ & $84 \%$ \\
\hline Moyenmoutier & $48 \%$ & $75 \%$ & $86 \%$ \\
\hline Raon l'Étape & $29 \%$ & $71 \%$ & $84 \%$ \\
\hline La Trouche & $39 \%$ & $72 \%$ & $84 \%$ \\
\hline Lunéville & $22 \%$ & $71 \%$ & $84 \%$ \\
\hline Autrey & $40 \%$ & $73 \%$ & $85 \%$ \\
\hline Gerbéviller & $31 \%$ & $67 \%$ & $81 \%$ \\
\hline Damelevières & $26 \%$ & $70 \%$ & $83 \%$ \\
\hline Malzéville & $25 \%$ & $71 \%$ & $84 \%$ \\
\hline
\end{tabular}

Le rapport entre le débit $Q 1 / 2$ et le module est extrêmement variable d'une station à l'autre. On peut toutefois effectuer des regroupements :

- pour les bassins situés dans les grès du Trias (stations de Moyenmoutier, La Trouche et Autrey Sainte-Hélène) le rapport est de $40 \%$ et plus. Ceci dénote la part importante de l'alimentation des écoulements par la nappe des grès ;

- pour le bassin de la Meurthe à Fraize, dans les Vosges cristallines, ce rapport est moins élevé $(35 \%)$. Ceci est dû à une plus forte proportion du ruissellement lié aux précipitations importantes du massif. Mais ce rapport n'est plus que de $25 \%$ à la station 
de Saint-Dié du fait du médiocre réservoir que constituent les grès argileux du Permien dans la dépression de Saint-Dié ;

- pour les bassins du Plateau lorrain, les rapports sont plus faibles : 31 \% à Gerbéviller sur la Mortagne qui bénéficie encore du soutien des grès du Trias à l'amont (Autrey Sainte-Hélène), mais seulement $22 \%$ à Lunéville sur la Vezouze où les affleurements des grès sont plus restreints ;

- pour l'ensemble du bassin de la Meurthe, à Damelevières ou à Malzéville, le rapport entre le débit $\mathrm{Q} 1 / 2$ et le module se stabilise autour de $25 \%$, ce qui montre que l'écoulement global de la Meurthe bénéficie d'un soutien non négligeable des nappes du Massif vosgien en étiage moyen.

Les rapports entre les débits de fréquence $1 / 2$ et $1 / 5$, puis entre les débits de fréquence $1 / 5$ et $1 / 10$, donnent des valeurs quasi identiques pour toutes les stations : 71 à $73 \%$ pour les premiers et 83 à $85 \%$ pour les seconds. Ceci est lié à l'écart-type des logarithmes des débits d'étiage qui est quasiment constant sur l'ensemble des cours d'eau du bassin de la Meurthe (tableau 4). Ces rapports traduisent une diminution lente et progressive des écoulements en étiage qui sont alimentés uniquement par la vidange des nappes souterraines. Les rapports sont un peu plus élevés à Fraize (Haute-Meurthe) et à Moyenmoutier (Rabodeau), ce qui s'explique par une vidange plus lente d'aquifères mieux alimentés. Par contre, ces rapports sont légèrement plus faibles à Gerbéviller (Mortagne) du fait d'une vidange plus rapide de certains aquifères, et en particulier celui des calcaires karstifiés du Muschelkalk.

Tableau 4 : Valeur de l'écart-type de l'ajustement à la loi log-normale

\begin{tabular}{|c|c|}
\hline Station & Écart-type \\
\hline Fraize & 0,328 \\
\hline Saint-Dié & 0,402 \\
\hline Moyenmoutier & 0,347 \\
\hline Raon l'Étape & 0,405 \\
\hline La Trouche & 0,39 \\
\hline Lunéville & 0,402 \\
\hline Autrey & 0,379 \\
\hline Gerbéviller & 0,472 \\
\hline Damelevières & 0,422 \\
\hline Malzéville & 0,402 \\
\hline
\end{tabular}

Il y a donc, pour ces débits caractéristiques d'étiage, une évolution assez homogène sur l'ensemble du bassin. Cependant, cela ne veut pas dire que cette évolution s'effectue dans le même laps de temps pour toutes les stations. En effet, la vidange de la nappe des 
grès est généralement plus lente que celle des calcaires karstifiés du Muschelkalk. Il peut en résulter ainsi un décalage de plusieurs jours, voire d'un mois, entre deux stations pour que la même fréquence soit atteinte.

\section{Comparaison des débits caractéristiques entre les stations}

Si l'évolution entre les débits d'étiage ( $Q 1 / 2, Q 1 / 5, Q 1 / 10)$ est identique entre deux stations, il en résulte nécessairement un rapport unique pour les différentes fréquences (tableau 5). C'est le cas par exemple de la station de Saint-Dié qui est bien corrélée avec celle de Raon l'Étape (rapport de $50 \%$ ) ou avec celle de Lunéville (rapport de $66 \%$ ). Par contre, si l'évolution n'est pas la même, les rapports sont variables pour les différentes stations. Ainsi, la station de Saint-Dié est moins bien corrélée avec celle de Fraize (rapport de 34 à $37 \%$ ) ou avec celle de Gerbéviller (rapport de 82 à $75 \%$ ). Dans ces deux cas, les rapports sont non seulement différents mais en plus ils ne varient pas dans le même sens.

Tableau 5 : Rapport entre les débits caractéristiques des différentes stations

\begin{tabular}{|c|c|c|c|c|c|c|c|c|c|c|c|c|c|c|c|c|c|c|c|c|}
\hline & \multicolumn{4}{|c|}{ Fraize } & \multicolumn{4}{|c|}{ Saint-Dié } & \multicolumn{4}{|c|}{ Moyenmoutier } & \multicolumn{4}{|c|}{ Raon l'Étape } & \multicolumn{4}{|c|}{ La Trouche } \\
\hline & Module & $\mathrm{Q}^{1 / 2}$ & Q1/5 & Q1/10 & Module & $Q_{1 / 2}$ & Q1/5 & Q1/10 & Module & Q1/2 & Q1/5 & Q1/10 & Module & Q 1/2 & Q1/5 & Q1/10 & Module & Q1/2 & Q1/5 & Q 1/10 \\
\hline Fraize & & & & & $27 \%$ & $34 \%$ & $36 \%$ & $37 \%$ & $79 \%$ & $58 \%$ & $59 \%$ & $59 \%$ & $14 \%$ & $17 \%$ & $18 \%$ & $19 \%$ & $91 \%$ & $83 \%$ & $87 \%$ & $90 \%$ \\
\hline Saint-Dié & & & & & & & & & $34 \%$ & $59 \%$ & $61 \%$ & $63 \%$ & $53 \%$ & $50 \%$ & $50 \%$ & $50 \%$ & $29 \%$ & $41 \%$ & $41 \%$ & $42 \%$ \\
\hline Moyenmoutier & & & & & & & & & & & & & $18 \%$ & $29 \%$ & $31 \%$ & $32 \%$ & $87 \%$ & $70 \%$ & $67 \%$ & $66 \%$ \\
\hline Raon l'Étape & & & & & & & & & & & & & & & & & $16 \%$ & $20 \%$ & $21 \%$ & $21 \%$ \\
\hline La Trouche & & & & & & & & & & & & & & & & & & & & \\
\hline Lunéville & & & & & & & & & & & & & & & & & & & & \\
\hline Autrey & & & & & & & & & & & & & & & & & & & & \\
\hline Gerbéviller & & & & & & & & & & & & & & & & & & & & \\
\hline Damelevières & & & & & & & & & & & & & & & & & & & & \\
\hline Malzéville & & & & & & & & & & & & & & & & & & & & \\
\hline
\end{tabular}

\begin{tabular}{|c|c|c|c|c|c|c|c|c|c|c|c|c|c|c|c|c|c|c|c|c|}
\hline & \multicolumn{4}{|c|}{ Lunéville } & \multicolumn{4}{|c|}{ Autrey Sainte-Hélène } & \multicolumn{4}{|c|}{ Gerbéviller } & \multicolumn{4}{|c|}{ Damelevières } & \multicolumn{4}{|c|}{ Malzéville } \\
\hline & Module & Q1/2 & Q1/5 & Q1/10 & Module & Q1/2 & Q $1 / 5$ & Q 1/10 & Module & Q1/2 & Q1/5 & Q1/10 & Module & Q $1 / 2$ & Q1/5 & Q1/10 & Module & Q1/2 & Q1/5 & Q 1/10 \\
\hline Fraize & $32 \%$ & $52 \%$ & $55 \%$ & $57 \%$ & $81 \%$ & $93 \%$ & $89 \%$ & $87 \%$ & $36 \%$ & $41 \%$ & $47 \%$ & $50 \%$ & $6 \%$ & $8 \%$ & $9 \%$ & $9 \%$ & $5 \%$ & $8 \%$ & $8 \%$ & $8 \%$ \\
\hline Saint-Dié & $83 \%$ & $66 \%$ & $66 \%$ & $66 \%$ & $22 \%$ & $32 \%$ & $32 \%$ & $33 \%$ & $74 \%$ & $82 \%$ & $77 \%$ & $75 \%$ & $23 \%$ & $25 \%$ & $25 \%$ & $25 \%$ & $20 \%$ & $22 \%$ & $22 \%$ & $22 \%$ \\
\hline Moyenmoutier & $41 \%$ & $89 \%$ & $93 \%$ & $95 \%$ & $64 \%$ & $54 \%$ & $53 \%$ & $52 \%$ & $46 \%$ & $71 \%$ & $79 \%$ & $84 \%$ & $8 \%$ & $14 \%$ & $15 \%$ & $16 \%$ & $7 \%$ & $13 \%$ & $14 \%$ & $14 \%$ \\
\hline Raon l'Étape & $44 \%$ & $33 \%$ & $33 \%$ & $33 \%$ & $12 \%$ & $16 \%$ & $16 \%$ & $16 \%$ & $39 \%$ & $41 \%$ & $39 \%$ & $37 \%$ & $44 \%$ & $49 \%$ & $50 \%$ & $50 \%$ & $37 \%$ & $44 \%$ & $44 \%$ & $44 \%$ \\
\hline La Trouche & $35 \%$ & $62 \%$ & $63 \%$ & $63 \%$ & $74 \%$ & $77 \%$ & $78 \%$ & $78 \%$ & $40 \%$ & $50 \%$ & $53 \%$ & $55 \%$ & $7 \%$ & $10 \%$ & $10 \%$ & $11 \%$ & $6 \%$ & $9 \%$ & $9 \%$ & $9 \%$ \\
\hline Lunéville & & & & & $26 \%$ & $48 \%$ & $49 \%$ & $49 \%$ & $78 \%$ & $80 \%$ & $85 \%$ & $88 \%$ & $19 \%$ & $16 \%$ & $17 \%$ & $17 \%$ & $16 \%$ & $15 \%$ & $15 \%$ & $15 \%$ \\
\hline Autrey & & & & & & & & & $29 \%$ & $38 \%$ & $42 \%$ & $43 \%$ & $5 \%$ & $8 \%$ & $8 \%$ & $8 \%$ & $4 \%$ & $7 \%$ & $7 \%$ & $7 \%$ \\
\hline Gerbéviller & & & & & & & & & & & & & $17 \%$ & $20 \%$ & $19 \%$ & $19 \%$ & $15 \%$ & $18 \%$ & $17 \%$ & $17 \%$ \\
\hline Damelevières & & & & & & & & & & & & & & & & & $86 \%$ & $90 \%$ & $88 \%$ & $87 \%$ \\
\hline Malzéville & & & & & & & & & & & & & & & & & & & & \\
\hline
\end{tabular}

NB : les rapports sont calculés en prenant en compte les débits les plus faibles par rapport aux plus élevés.

Globalement les stations de Moyenmoutier et de Fraize offrent des rapports disparates avec les autres stations, mais elles sont bien corrélées entre elles. Seule la station de Gerbéviller n'a aucune corrélation bien nette avec une autre station.

Cependant, la différence entre les rapports ne dépasse guère les $5 \%$. 


\section{Cartographie des rendements}

42 L'utilisation des valeurs de rendement d'étiage aux stations hydrométriques, pour les différentes fréquences caractéristiques, peut permettre d'effectuer une cartographie de ces rendements. Mais avec 10 stations pour un bassin de $3085 \mathrm{~km} 2$, cette cartographie serait trop générale, d'où la nécessité d'utiliser les profils hydrologiques pour réaliser une cartographie plus détaillée.

\section{A. L'utilisation des profils hydrologiques}

Les profils hydrologiques permettent de connaître les débits tout au long d'un cours d'eau et d'en déduire pour tout tronçon de celui-ci les apports qui lui sont propres. Ce tronçon correspondant à une surface de bassin, on en déduit un débit spécifique qui caractérise le rendement particulier de ce bassin intermédiaire. On peut ainsi multiplier les bassins intermédiaires en prenant en compte l'organisation des écoulements (apports dus aux affluents, aux sources, au drainage de nappes, ...).

Le calage des profils hydrologiques sur les débits caractéristiques d'étiage des stations hydrométriques permet de qualifier les rendements fréquentiels des bassins intermédiaires à l'amont d'une station ou entre deux stations.

L'analyse comparée des débits fréquentiels des différentes stations a montré que les rapports de ces débits se caractérisaient par une valeur unique ou des valeurs proches (variation de $5 \%$ au maximum). On peut donc considérer que l'ensemble du bassin de la Meurthe est relativement homogène dans l'évolution des modalités d'écoulement en étiage. La cartographie des rendements d'étiage caractéristiques permet de suivre l'évolution spatiale de ceux-ci.

Pour cette cartographie, les rendements sont déterminés pour les zones définies par la "codification hydrographique» du bassin Rhin-Meuse qui couvrent des surfaces comparables de $30 \mathrm{~km} 2$ en moyenne. Les classes des débits sont définies en fonction des rendements moyens d'étiage des différentes formations aquifères (Fig. 4). 
Fig. 4 : Bassin de la Meurthe ; cartes des rendements

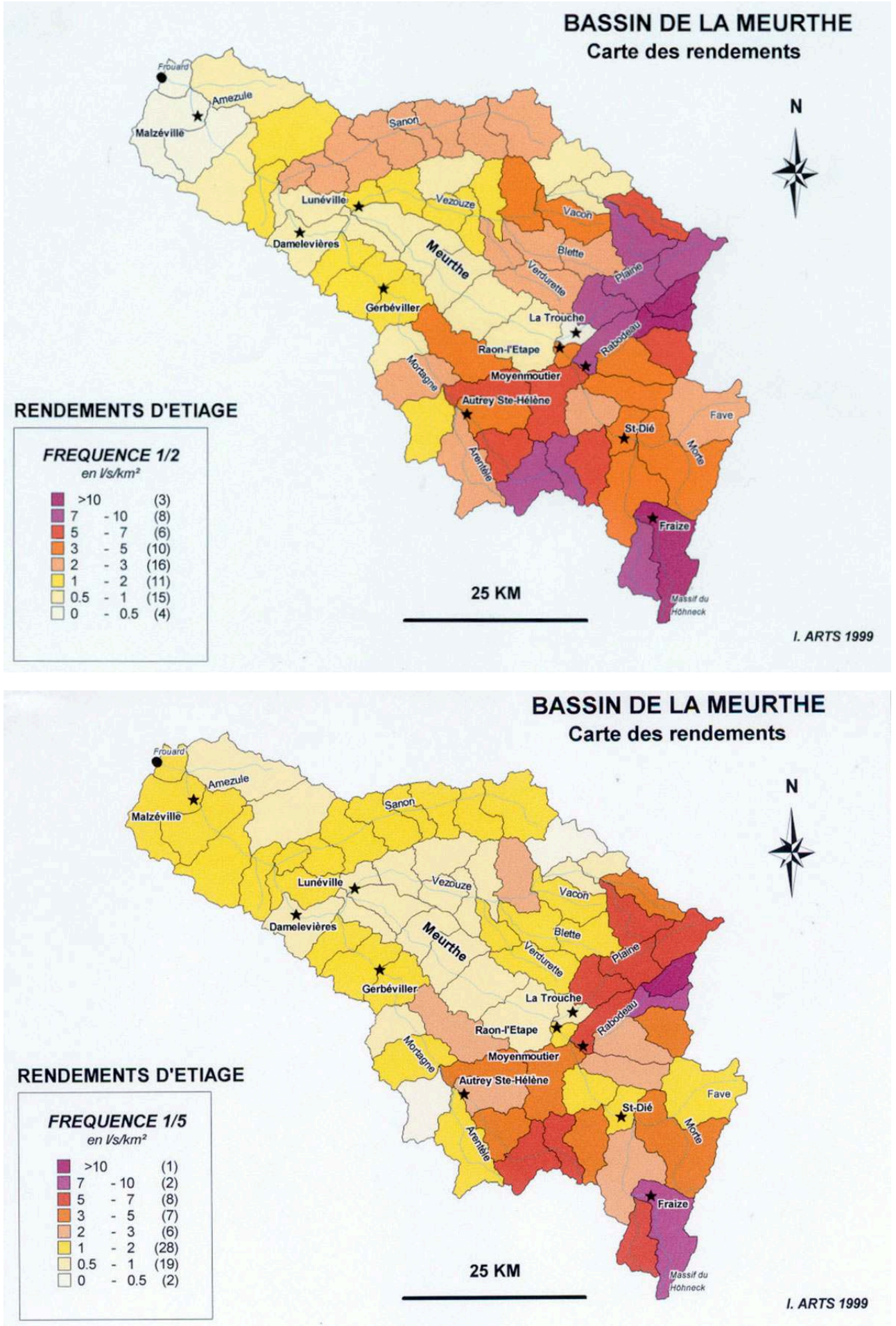




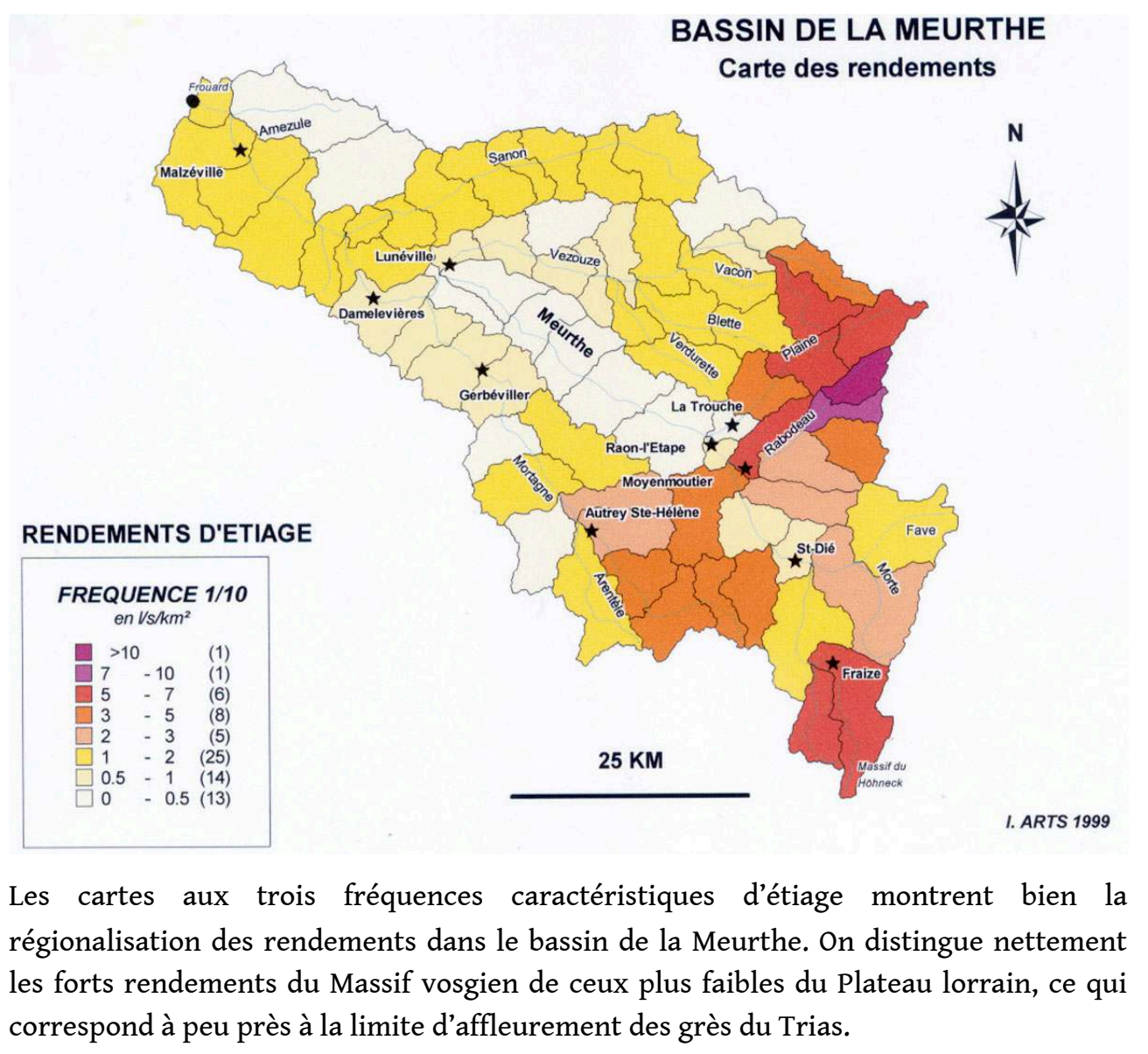

\section{B. Le Massif vosgien}

Les zones à haut rendement se situent dans les formations cristallines et métamorphiques du massif (bassins de la Haute Meurthe et de la Morte) et dans les formations gréseuses du Trias (bassins du Rabodeau, de la Plaine, de la Mortagne amont et de la Vezouze amont). Cependant, une zone intermédiaire est moins productive (bassin de la Fave et dépression de Saint-Dié).

L'évolution entre les rendements des trois cartes est différente. Entre les fréquences $1 / 2$ et $1 / 5$, les rendements diminuent rapidement alors qu'entre les fréquences $1 / 5$ et $1 / 10$ la diminution est moins rapide. Il y a donc une stabilisation des rendements vers les hautes fréquences, ce qui démontre que certains aquifères ont de fortes capacités.

Les zones les plus productives se situent d'une part à l'extrême amont de la Meurthe, là où le socle cristallin, très fracturé, comporte d'importantes poches d'altérites et où le fond de la vallée est encombré de puissants dépôts glaciaires, et d'autre part, à l'amont des bassins du Rabodeau, de la Plaine et de la Vezouze, là où la couverture des grès du Trias est plus épaisse. Le bassin amont du Rabodeau conserve un rendement toujours élevé (supérieur à $7 \mathrm{l} / \mathrm{s} / \mathrm{km} 2$ ) qui peut s'expliquer par un transfert de nappe des bassins gréseux situés à l'est, sur le versant alsacien, où on observe en effet des rendements beaucoup plus faibles. La vidange des grès du bassin amont de la Mortagne (station d'Autrey Sainte-Hélène) est un peu plus importante du fait de la moindre épaisseur des grès du Trias. 
51 Les zones les moins productives se concentrent dans le bassin permien de Saint-Dié (grès argileux) et dans le bassin de la Fave où les formations gneissiques compactes constituent un réservoir de faible capacité. Ces zones, dont les rendements ne dépassent pas $5 \mathrm{l} / \mathrm{s} / \mathrm{km} 2$ pour la fréquence $1 / 2$, passent progressivement à des rendements inférieurs à $2 \mathrm{l} / \mathrm{s} / \mathrm{km} 2$ (fréquence $1 / 5$ ) et même inférieurs à $1 \mathrm{l} / \mathrm{s} / \mathrm{km} 2$ (fréquence 1/10). Ces rendements sont comparables à ceux du Plateau lorrain où les aquifères sont peu productifs.

52 Les rendements sont donc contrastés dans le Massif vosgien et ces contrastes perdurent entre les trois fréquences. Ceci s'explique par la diversité des capacités aquifères des formations et leur fracturation, mais aussi par des transferts entre bassins au niveau des interfluves (limite hydrogéologique difficile à déterminer) et par des circulations sous-alluviales dans les dépôts glaciaires et fluvio-glaciaires de fond de vallée.

\section{Le Plateau lorrain}

53 Les rendements dépassent rarement $3 \mathrm{l} / \mathrm{s} / \mathrm{km} 2$ et sont moins contrastés. Cette plus grande homogénéité des rendements est due aux faibles capacités aquifères des formations sédimentaires à dominante argileuse. Les calcaires du Muschelkalk peuvent localement produire des rendements un peu plus élevés, et les grès du Rhétien, situés à l'aval du bassin, soutenir légèrement les étiages des affluents de la Meurthe entre Damelevières et Malzéville.

54 Comme précédemment dans le Massif vosgien, la décroissance des rendements est surtout sensible entre les fréquences $1 / 2$ et $1 / 5$. Ensuite, la diminution des rendements est faible et peu perceptible, même en utilisant des classes de débit aux limites très rapprochées.

55 Les zones les plus productives (rendements supérieurs à $2 \mathrm{l} / \mathrm{s} / \mathrm{km} 2$ ), ne sont présentes que pour la fréquence $1 / 2$. Elles sont localisées principalement sur les calcaires du Muschelkalk, au contact des grès du Trias, et plus particulièrement sur les affluents de rive gauche de la Vezouze, là où les calcaires, plus soulevés par la tectonique, permettent un meilleur recoupement de la nappe par les cours d'eau.

À cette fréquence, le bassin du Sanon présente également, pour toutes les zones hydrographiques, un rendement supérieur à $2 \mathrm{l} / \mathrm{s} / \mathrm{km} 2$, alors qu'il est inscrit principalement dans les marnes du Keuper. Dans ce bassin, les rendements naturels, qui devraient être plus faibles, sont influencés par le canal de la Marne-au-Rhin dont le tracé est parallèle au cours du Sanon. Les apports du canal (déversoirs de trop-plein, fuites à travers les digues) étant difficiles à déterminer, il n'est pas possible de reconstituer les débits naturels. Les zones situées entre Damelevières et Malzéville sont également soumises à ces influences.

Pour les autres fréquences $(1 / 5$ et $1 / 10)$, ces zones productives ont des rendements plus faibles, mais toujours supérieurs à $1 \mathrm{l} / \mathrm{s} / \mathrm{km} 2$.

Les zones les moins productives, à la fréquence $1 / 2$, sont les bassins aval de la Vezouze et de la Mortagne (rendements inférieurs à $2 \mathrm{l} / \mathrm{s} / \mathrm{km} 2$ ) inscrits dans les marnes du Keuper, et le bassin de la Meurthe entre la confluence de la Plaine et celle de la Vezouze (rendements inférieurs à $1 \mathrm{l} / \mathrm{s} / \mathrm{km} 2$ ) qui n'est alimenté que par la nappe alluviale (aucun affluent et aucune source). 

encore soutenus pour les cours d'eau qui entaillent les calcaires du Muschelkalk ou pour ceux qui sont sous influence anthropique (apports du canal). Les rendements sont plus indigents pour les cours d'eau qui s'inscrivent essentiellement dans les marnes du Keuper.

61 Cette analyse de la cartographie des rendements en étiage du bassin de la Meurthe montre bien que ceux-ci dépendent essentiellement du potentiel aquifère des formations recoupées par les différents cours d'eau. L'évolution entre les trois fréquences $(1 / 2,1 / 5$ et $1 / 10)$ permet de caractériser la vidange plus ou moins prononcée des différentes nappes souterraines.

\section{Conclusion}

La connaissance des débits de basses-eaux dans le bassin de la Meurthe, qu'ils soient observés aux stations hydrométriques ou mesurés lors de campagnes de jaugeages, permet de comprendre les modalités de l'écoulement dans ce bassin divisé en deux secteurs bien distincts. Grâce à la réalisation des profils hydrologiques, on peut spatialiser des rendements d'étiage et caractériser ainsi les comportements hydrologiques des sous-bassins. Toutefois, la spatialisation présentée ici est limitée par l'utilisation des zones déterminées par la "codification hydrographique " du bassin Rhin-Meuse. Une autre cartographie, respectant mieux les ruptures de pente des profils hydrologiques, permet de spatialiser les écoulements de façon beaucoup plus adaptée à la sectorisation des zones hydrogéologiques homogènes des différents bassins. C'est le cas également pour les cartes réalisées en mode maillé (ou « raster ») qui donnent une valeur de débit par km2. Le CEGUM améliore les modes de représentation et de spatialisation des écoulements en basses eaux dans le but de réaliser une cartographie globale de ces phénomènes pour les bassins de la Moselle et de la Meuse.

\section{BIBLIOGRAPHIE}

Arts I., François D., 1997. - Profils hydrologiques du bassin de la Meurthe - Proposition d'une typologie - Mosella, Tome XXIII, $\mathrm{n}^{\circ}$ 1-2, pp. 165-178.

Arts I., François D., Corbonnois J., Sary M., 1996. - Réalisation d'un catalogue des débits caractéristiques des cours d'eau du bassin de la Meurthe - Rapport CEGUM - Agence de l'Eau RhinMeuse, 30 p., annexes.

Decloux J.P., Sary M., 1991. - Campagnes d'étiage. Objectifs, traitement et valorisation des données - Mosella, Tome XVIII, $\mathrm{n}^{\circ}$ spécial annuel, pp. 121-134.

Revue Géographique de l'Est, vol. 40 / 1-2 | 2000 
François D., Sary M., Auer J.-C., Zumstein J.-F. (1994). - Étude méthodologique des débits d'étiage Rapport CEGUM - Agence de l'Eau Rhin-Meuse, $30 \mathrm{p}$.

\section{RÉSUMÉS}

Les campagnes de jaugeages en basses eaux permettent de faire une investigation spatiale des écoulements à l'intérieur des bassins et d'élaborer les profils hydrologiques des différents cours d'eau qui servent à la détermination des zones de rendement homogène. Ces profils sont calés sur les débits fréquentiels caractéristiques des stations hydrométriques. Une carte des rendements hydrologiques pour l'ensemble du bassin est alors réalisée pour chacune des fréquences choisies.

Measures of low water conditions facilitate a spatial investigation of flows within river basins and the construction of hydrological profiles of different waterways which can be used to determine zones of homogeneous flows. These profiles are fixed on characteristics flow frequencies of hydrological stations. A map of hydrological outputs for the whole basin is then produced for each of the selected frequencies.

Die Messkampagnen bei Niedrigwasser ermöglichen eine räumliche Erforschung der Niderschläge im Inneren der Becken und die Ausarbeitung hydrologischer Profile der verschiedenen Wasserläufe, die zur Bestimmung von Zonen homogener Ergiebigkeit für das ganze Becken und für der ausgewählten Häufigkeiten dargestellt.

\section{INDEX}

Keywords : cartography, hydrological profile, low water flows, Meurthe, output

Mots-clés : cartographie, étiage, Meurthe, profil hydrologique, rendement

Schlüsselwörter : Ergiebigkeit, hydrologisches Profil, Kartographie, Meurthe, Niedrigwasser

\section{AUTEURS}

\section{ISABELLE ARTS}

Centre d'Études Géographiques de l'Université de Metz (CEGUM) - Laboratoire de Géographie Physique - UFR Sciences Humaines et Arts - Ile du Saulcy, 57045 Metz Cedex 1

\section{MICHEL SARY}

Centre d'Études Géographiques de l'Université de Metz (CEGUM) - Laboratoire de Géographie Physique - UFR Sciences Humaines et Arts - Ile du Saulcy, 57045 Metz Cedex 1 